\title{
PREVALÊNCIA DE SOBREPESO E OBESIDADE EM PRÉ-ESCOLARES DE ALTO NÍVEL SÓCIO- ECONÔMICO DA CIDADE DE ARACAJU-SE
}

\author{
PREVALENCE OF OVERWEIGHT AND OBESITY AMONG PRESCHOOL CHILDREN \\ OF HIGH LEVEL ECONOMICAL PARTNER OF ARACAJU-SE
}

Diego A. S. Silva

Mestrando em Educação Física da Universidade Federal de Santa Catarina. Núcleo de Cineantropometria e Desempenho Humano. Núcleo de Pesquisa em Aptidão Física de Sergipe.

CorRespondência: Diego Augusto Santos Silva. Avenida Gonçalo Rollemberg Leite, n 1960, Condomínio Alphaville I, Ed. Gama, Apto 1201, Bairro Suissa, Aracaju-Sergipe. CEP: 49050370. E-mail: diegoaugustoss@yahoo.com.br

Silva DAS. Prevalência de sobrepeso e obesidade em pré-escolares de alto nível sócioeconômico da cidade de Aracaju-SE. Medicina (Ribeirão Preto) 2008; 41 (2): 177-81.

RESUMO: Modelo de Estudo: Estudo de Prevalência. Objetivo: O objetivo do presente estudo foi estimar a prevalência de sobrepeso e obesidade em pré-escolares de alto nível socioeconômico da cidade de Aracaju-Sergipe, Brasil, medido de forma direta. Métodos: 243 crianças, sendo $51 \%$ do sexo feminino de 3,5 a 5,5 anos de idade de alto nível socioeconômico, segundo critérios da Associação Nacional de Empresa de Pesquisa, foram avaliadas quanto ao peso e a estatura, para se calcular o IMC e classificá-las em normal, sobrepeso ou obesidade de acordo com os critérios de Cole et al. Resultados: Na amostra estudada a prevalência de sobrepeso foi de $16 \%$ e a obesidade de $7,9 \%$, sendo que não houve diferenças significativas na prevalência de sobrepeso e obesidade entre meninos e meninas $(p>0,05)$. Não houve também diferenças entre os grupos de idade para cada sexo $(p>0,05)$. Conclusão: $O$ sobrepeso e a obesidade se fizeram presentes em uma amostra de crianças de 3,5 a 5,5 anos de idade de alto poder aquisitivo da cidade de Aracaju-SE.

Descritores: Obesidade. Pré-escolar. Estudos Transversais. Classe Social. Saúde da Criança.

\section{1- INTRODUÇÃO}

Nos últimos anos, o aumento do sobrepeso e obesidade em crianças, adolescentes e adultos vem se tornando um problema de saúde global. No Brasil, estudos têm indicado que para a população geral a prevalência de obesidade mantém-se estável, em torno de $12-13 \%{ }^{1,2}$.

As taxas de sobrepeso e obesidade na infância e adolescência ao redor do mundo são consideradas altas. No Canadá, por exemplo, a prevalência combinada de sobrepeso e obesidade em meninos e meninas com idade escolar era de 15\% em 1981 e, passou para $35,4 \%$ em $1996^{3}$. Na Austrália, dados coletados em 1985 e 1995 com pessoas de sete a quinze anos de idade demonstraram que, a prevalência de excesso de peso passou de $10,7 \%$ para $20 \%$ entre os garotos e, de $11,8 \%$ para $21,5 \%$ entre as garotas ${ }^{4}$. Nos Estados Unidos, aumentos similares no excesso de peso foram reportados no mesmo período, sendo que em crianças de dois a cinco anos de idade esta prevalência era de 7,2\%. Em escolares de seis a onze anos de idade a prevalência chegava a $11,3 \%$ e, dos doze aos dezenove anos era de $10,5 \%{ }^{5}$. Ogden et al. ${ }^{6}$ com dados de 1999-2000, na população de crianças e adolescentes norte-americana, comenta que as taxas de ex- 
cesso de peso tenderam a aumentar, sendo $10,4 \%$, $15,3 \%$ e $15,5 \%$ respectivamente, para as idades supracitadas.

Poucos estudos nacionais têm sido publicados levando-se em consideração a prevalência de sobrepeso e obesidade na população pré-escolar, contudo, estes poucos achados indicam também que há uma tendência a um aumento de peso nesta idade. Já nos países desenvolvidos muitas pesquisas mostram esse cenário. No Canadá, por exemplo, em um estudo publicado em 2001 com dados da década passada, diagnosticou-se que aproximadamente $20 \%$ de crianças em idade pré-escolar tinham excesso de peso ${ }^{7}$. No Brasil, comparando-se os dados do Estudo Nacional da Despesa Familiar (ENDEF), realizado em 1974/75, com os dados da Pesquisa sobre Padrões de Vida (PPV), realizada em 1996/97 somente nas regiões Sudeste e Nordeste verificou-se um aumento na prevalência de sobrepeso e obesidade de $4,1 \%$ para $13,9 \%$ em crianças e adolescentes de seis a dezoito anos ${ }^{8}$. Considerando que há um aumento nesta prevalência, tanto em países desenvolvidos como nos em desenvolvimento e, a escassez de dados desta natureza com crianças de Aracaju-SE, torna-se de fundamental importância estabelecer esta estimativa com uma amostra desta região.

Sendo assim, o objetivo do nosso estudo é determinar a prevalência de sobrepeso e obesidade em pré-escolares de alto nível socioeconômico da cidade de Aracaju-Sergipe, Brasil. O estudo torna-se pioneiro no estado de Sergipe, à medida que não se encontrou na literatura estudos semelhantes com estas características realizados no estado.

\section{2- MATERIAIS E MÉTODOS}

O estudo de caráter transversal teve como população crianças de 3,5 a 5,5 anos matriculadas em três escolas particulares da cidade de Aracaju-SE. Escolheu-se as três escolas porque são as que possuem as maiores mensalidades para crianças nesta faixa-etária, tornando-se, em tese, as escolas mais procuradas pelas classes sociais mais altas. Todavia, vale ressaltar que, estas três escolas e os seus alunos não são uma parte representativa dos estabelecimentos de ensino da cidade de Aracaju.

O número total de crianças nesta faixa-etária dos três estabelecimentos de ensino somava-se 363 crianças. Contudo, para o presente estudo, que visava somente crianças de alto nível socioeconômico, fez-se necessário identificar quantas tinham estas características. Para isto, os pais ou responsáveis dos alunos foram contactados e explicados dos objetivos do estudo, tendo que assinar um "Termo de Consentimento Livre e Esclarecido" para permitir a participação dos filhos na pesquisa. Após assinar o termo, os pais ou responsáveis tinham que preencher um questionário referente a nível socioeconômico9 visando uma homogeneidade da amostra quanto à classe socioeconômica.

Os critérios de classificação econômica do Brasil, proposta pela ANEP ${ }^{9}$, classifica em cinco classes econômicas (A,B,C,D e E). A classe A corresponde a de maior poder aquisitivo e, a $\mathrm{E}$ de menor poder. Para a presente pesquisa, os indivíduos classificados nas classes A e B foram agrupados e tratados como de maior poder aquisitivo, por entender que, na cidade de Aracaju há pouca diferença entre essas duas classes.

Pelo fácil e livre acesso às escolas e aos alunos optou-se por coletar os valores de peso e estatura de todas as crianças em idade pré-escolar. Das 363 crianças nesta idade, 252 somaram-se como de maior poder aquisitivo, formando assim a amostra da presente pesquisa, sendo 130 do sexo feminino e 122 do masculino.

No dia da coleta de dados referentes ao peso e a estatura, nove crianças faltaram, sendo então a amostra formada por 243 pré-escolares. Destes, 124 (51\%) era do sexo feminino e 119 (49\%) do sexo masculino.

Foram coletados dados de peso, por meio de uma balança digital da marca Tech Line ${ }^{\circledR}$, com precisão de 100 gramas. E a estatura com um estadiômetro profissional portátil da marca Sanny®, com precisão de um milímetro. As coletas seguiram as padronizações descritas em Petroski ${ }^{10}$. Além destes, foi coletado a data de nascimento das crianças.

Dois acadêmicos do sétimo período do curso de Educação Física da Universidade Federal de Sergipe foram devidamente treinados pelo autor do presente estudo quanto às padronizações para a coleta dos dados.

Após a coleta destes dados, calculou-se o índice de massa corporal (IMC) por meio da fórmula $\left(\mathrm{Peso} /\right.$ Estatura $\left.^{2}\right)$. Após estes cálculos, os valores foram classificados de acordo com o sexo e com a idade em Normal, Sobrepeso ou Obesidade, segundo critérios de Cole et al. ${ }^{11}$. Este estudo ${ }^{11}$ determinou pontos de corte para o sobrepeso e obesidade em crianças e adolescentes, tendo como base, dados de seis 
países: Brasil, Grã Bretanha, Hong Kong, Holanda, Singapura e Estados Unidos. Sendo que, para a idade de 18 anos o IMC de $25 \mathrm{~kg} / \mathrm{m}^{2}$ é o ponto de corte para sobrepeso e o de $30 \mathrm{~kg} / \mathrm{m}^{2}$ é o de obesidade, em ambos os sexos.

O quadro a seguir diz respeito aos pontos de corte de Cole et al. ${ }^{11}$ para a faixa-etária da presente pesquisa.

\begin{tabular}{|c|c|c|c|c|}
\hline (anos) & Masculino & Feminino & Masculino & Feminino \\
\hline 3,5 & 17,69 & 17,40 & 19,39 & 19,23 \\
\hline 4 & 17,55 & 17,28 & 19,29 & 19,15 \\
\hline 4,5 & 17,47 & 17,19 & 19,26 & 19,12 \\
\hline 5 & 17,42 & 17,15 & 19,30 & 19,17 \\
\hline 5,5 & 17,45 & 17,20 & 19,47 & 19,34 \\
\hline
\end{tabular}

Os dados foram analisados usando o pacote estatístico SPSS 10.0 for Windows. Os tratamentos estatísticos empregados foram a estatística descritiva e o teste qui-quadrado para identificar diferenças entre as proporções, considerando o nível de significância de $5 \%$.

\section{3- RESULTADOS}

A Tabela I descreve a amostra em relação ao peso, a estatura e o IMC de acordo com o sexo e a idade das crianças. A média do peso, da estatura e do
IMC nos garotos foi de $19,5 \mathrm{~kg}, 106,9 \mathrm{~cm}$ e $16,7 \mathrm{~kg} / \mathrm{m}^{2}$, respectivamente. Já nas garotas esses valores foram de $18,6 \mathrm{~kg}, 106,3 \mathrm{~cm}$ e $16,4 \mathrm{~kg} / \mathrm{m}^{2}$, respectivamente.

$\mathrm{Na}$ Tabela II, pode-se observar a prevalência de sobrepeso e obesidade de acordo com a idade e o sexo. Verificou-se que o sobrepeso e a obesidade se fizeram presentes em todas as faixas etárias, tendo o sobrepeso uma prevalência de $16 \%$ e a obesidade de $7,9 \%$ na amostra estudada. Não houve diferenças significativas na prevalência de sobrepeso e obesidade entre meninos e meninas ( $p>0,05)$. Não houve também diferenças entre os grupos de idade para cada $\operatorname{sexo}(p>0,05)$.

\section{4- DISCUSSÃO}

A obesidade é relatada como um problema de ordem pública por órgãos internacionais ${ }^{5}$. Embora, o presente estudo não tenha usado uma amostra representativa da cidade e, inferências maiores não possam ser tiradas, ao que consta, este é o primeiro estudo com estas características no estado de Sergipe, podendo-se observar por meio dos resultados que o sobrepeso e a obesidade se fizeram presentes em todas as idades analisadas.

A prevalência nacional de sobrepeso, em crianças menores de cinco anos de idade, detectada pela Pesquisa Nacional sobre Demografia e Saúde ${ }^{12}$, foi de $4,9 \%$, sendo que os dados do presente estudo foram maiores do que os apresentados em 1996.

A prevalência de excesso de peso (sobrepeso + obesidade) no presente estudo foi de $23,9 \%$, muito superior à verificada em outros locais do Brasil com

Tabela I. Valores médios e o desvio padrão do Peso, Estatura e IMC de acordo com o sexo e a idade em meses dos pré-escolares de Aracaju-SE em 2007.

\begin{tabular}{|c|c|c|c|c|c|}
\hline \multirow[b]{2}{*}{ Sexo/Variáveis } & \multicolumn{4}{|c|}{ Grupos de idade (meses) } & \multirow[b]{2}{*}{ Toda Amostra } \\
\hline & $42-48$ & $49-54$ & $55-60$ & $61-66$ & \\
\hline Masculino & $\mathrm{n}=30$ & $\mathrm{n}=32$ & $\mathrm{n}=29$ & $\mathrm{n}=28$ & $\mathrm{n}=119$ \\
\hline Peso (kg) & $18,3(3,1)$ & $19,0(3,1)$ & $19,5(3,2)$ & $20,3(4,3)$ & $19,5(3,2)$ \\
\hline Estatura $(\mathrm{cm})$ & $104,2(4,4)$ & $106,4(4,6)$ & $108,3(4,5)$ & $110,4(5,3)$ & $106,9(4,9)$ \\
\hline $\mathrm{IMC}\left(\mathrm{kg} / \mathrm{m}^{2}\right)$ & $16,8(2,1)$ & $16,8(1,9)$ & $16,6(1,9)$ & $16,7(2,0)$ & $16,7(2,0)$ \\
\hline Feminino & $\mathrm{n}=32$ & $\mathrm{n}=30$ & $\mathrm{n}=28$ & $\mathrm{n}=34$ & $\mathrm{n}=124$ \\
\hline Peso (kg) & $17,6(2,9)$ & $18,0(3,2)$ & $19,0(3,6)$ & $19,5(3,8)$ & $18,6(3,3)$ \\
\hline Estatura $(\mathrm{cm})$ & $102,7(4,6)$ & $104,9(4,5)$ & $107,6(5,2)$ & $110,1(6,4)$ & $106,3(5,0)$ \\
\hline $\operatorname{IMC}\left(\mathrm{kg} / \mathrm{m}^{2}\right)$ & $16,6(2,2)$ & $16,4(2,0)$ & $16,4(2,4)$ & $16,1(1,9)$ & $16,4(2,1)$ \\
\hline
\end{tabular}


Tabela II. Prevalência de sobrepeso e obesidade de acordo com o sexo e faixa-etária, em meses, dos pré-escolares de Aracaju-SE em 2007.

\begin{tabular}{lccccc}
\hline & \multicolumn{5}{c}{ Grupos de idade (meses) } \\
Sexo & $42-48$ & $49-54$ & $55-60$ & $61-66$ & Toda Amostra \\
\hline Masculino & $\mathrm{n}=30$ & $\mathrm{n}=32$ & $\mathrm{n}=29$ & $\mathrm{n}=28$ & $\mathrm{n}=119$ \\
Sobrepeso (\%) & 16,7 & 15,6 & 14,0 & 17,9 & 16,1 \\
Obesidade (\%) & 6,6 & 6,2 & 10,3 & 7,1 & 7,6 \\
Feminino & $\mathrm{n}=32$ & $\mathrm{n}=30$ & $\mathrm{n}=28$ & $\mathrm{n}=34$ & $\mathrm{n}=124$ \\
Sobrepeso (\%) & 18,8 & 16,7 & 21,4 & 8,8 & 16,4 \\
Obesidade (\%) & 9,4 & 6,7 & 10,7 & 5,9 & 8,2 \\
\hline
\end{tabular}

crianças menores de cinco anos. No Centro-Oeste esta prevalência foi de $5,6 \%$, no Nordeste e Norte de $4,5 \%$, $6,7 \%$ na cidade do Rio de Janeiro, 5,5\% na cidade de São Paulo e 4,7\% no Sul do Brasil ${ }^{12}$. Na cidade de Salvador, Estado da Bahia, a prevalência encontrada por Assis e Barreto ${ }^{13}$, utilizando-se o índice peso para altura, foi de 3,9\%. Na cidade de Florianópolis, a prevalência de excesso de peso foi de $6,8 \%^{14}$. Vale destacar que, os resultados destas regiões não levaram em consideração o nível socioeconômico, sendo a amostra daqueles estudos muito maior que a do presente.

Um fato a destacar é a não padronização de critérios de referências para o diagnóstico do excesso de peso, pois os estudos supracitados utilizaram como critério o índice peso para altura ${ }^{15,16}$, diferentemente do utilizado para o presente estudo que foi os critérios de Cole et al. ${ }^{11}$ para o IMC. Então, se torna necessário uma padronização nestes critérios para comparações mais exatas, sobretudo, para a população brasileira.

Considerando estudos internacionais que utilizaram como critério os pontos de corte de Cole et al. ${ }^{11}$, sem dividir por nível socioeconômico, observou-se que no Reino Unido um estudo representativo reportou uma prevalência de sobrepeso de $23,6 \%$ em crianças de 2,9 a 4 anos de idade ${ }^{17}$. Já no Canadá, a prevalência de excesso de peso entre crianças de três a seis anos de idade foi de $25,6 \%{ }^{18}$.

Embora a amostra de todos estes estudos terem sido maiores que a do presente, muitos até significativa para a região em análise, observa-se que, os resultados da atual investigação tornam-se mais próximos a dados de estudos internacionais do que dos nacionais.

Uma justificativa para que a prevalência de excesso de peso no presente estudo tenha se aproximado, em porcentagem, das de países desenvolvidos é o fato da amostra investigada ser de crianças de alto nível socioeconômico, tendo talvez, rotinas, atividades e, um estilo de vida parecido ao das crianças de países desenvolvidos. Cabe, portanto, uma análise de todo o estilo de vida dos escolares de alto poder aquisitivo para uma melhor comparação com os hábitos diários de crianças de países desenvolvidos.

Não havendo estudos no estado de Sergipe acerca desta temática com uma amostra com estas características, torna-se difícil a comparação de resultados. Porém, ao observar os dados do estudo de Wang, Monteiro \& Popkin ${ }^{8}$ com os dados da Pesquisa sobre Padrões de Vida (PPV), realizada em 1996/97, com indivíduos de seis a dezoito anos, sem levar em consideração o nível socioeconômico, observa-se que, no presente estudo houve uma maior prevalência de sobrepeso e obesidade. É bem verdade que naquele estudo $^{8}$ a amostra foi representativa das regiões Sudeste e Nordeste do Brasil, no entanto, cabe um monitoramento das crianças que se apresentaram na presente investigação com excesso de peso, evitando problemas de saúde futuros. Além disso, investigações com amostras significativas não só do estado de Sergipe, mas, sobretudo de todo o território nacional são necessárias para um melhor entendimento desta enfermidade chamada obesidade.

Com o exposto pode-se concluir que o sobrepeso e a obesidade se fizeram presentes em uma amostra de crianças de 3,5 a 5,5 anos de alto poder aquisitivo da cidade de Aracaju-SE. Valores estes, superiores a de outras regiões do Brasil e, semelhantes à de países desenvolvidos. 
Silva DAS. Prevalence of overweight and obesity among preschool children of high level economical partner of Aracaju-SE. Medicina (Ribeirão Preto) 2008; 41 (2): 177-81.

ABSTRACT: Model of Study: Prevalence Study. Objective: The objective of the present study was to esteem the prevalence of overweight and obesity among preschool children of high level economical partner of Aracaju-Sergipe city, Brazil, measured in a direct way. Methods: 243 children, being $51 \%$ of the female sex from 3,5 to 5,5 years old of high level economical partner, in agreement to National Association of Company of Research was appraised with relationship to the weight and the stature, to calculate IMC and to classify them in normal, overweight or obesity in agreement with the criteria of it Cole et al. Results: The prevalence of overweight was $16 \%$ and the obesity of $7,9 \%$, and there were not significant differences in the prevalence of overweight and obesity between boys and girls $(p>0,05)$, as well as there were not differences among the groups of age for each sex $(p>0,05)$. Conclusion: The overweight and the obesity were made presents in a sample of children from 3,5 to 5,5 years of age of high purchasing power of the Aracaju city.

Keywords: Obesity. Preschool. Cross-sectional Study. Social Class. Child Health.

\section{REFERÊNCIAS}

1 - Brasil. Ministério do Planejamento, Orçamento e Gestão. Instituto Brasileiro de Geografia e Estatística. Pesquisa de Orçamento Familiar 2002-2003. Análise da Disponibilidade Domiciliar de Alimentos e do Estado Nutricional no Brasil. Rio de Janeiro: IBGE; 2004. p.40-76.

2 - Brasil. Instituto Nacional de Alimentação e Nutrição. Pesquisa Nacional Sobre Saúde e Nutrição de 1989. Condições nutricionais da população brasileira: adultos e Idosos. Brasília: INAN; 1991.

3 - Tremblay MS, Willms JD. Secular trends in the body mass index of Canadian children [published erratum appears in CMAJ 2001;164(7):970]. CMAJ 2000; 163(11): 1429-33.

4 - Magarey AM, Daniels LA, Boulton TJ. Prevalence of overweight and obesity in Australian children and adolescents: reassessment of 1985 and 1995 data against new standard international definitions. Med J Aust 2001; 174: 561-4.

5 - The Surgeon General's call to action to prevent and decrease overweight and obesity. Rockville (MD): US Department of Health and Human Services, Public Health Service, Office of the Surgeon General; 2001.

6 - Ogden C, Flegal K, Carroll M., Johnson C. Prevalence and trends in overweight among US children and adolescents, 1999-2000. JAMA 2002; 288 (14): 1728-32.

7 - Peters RV. Better beginnings, better futures: overview of lessons learned. Kingston (ON): Queen's University, Research Coordination Unit; 2001.

8 - Wang Y, Monteiro C, Popkin BM. Trends of obesity and underweight in older children and adolescents in the United States, Brazil, China, and Rússia. Am J Clin Nutr 2002; 75 : 971-7.
9 - ANEP - Associação Nacional de Empresa de Pesquisa. Critério de Classificação Econômica Brasil: São Paulo. www.anep.org.br/cceb.zip. Acesso em 3/12/2003. 2000

10 - Petroski EL. Antropometria: técnicas e padronizações. 3 ed. Blumenau: Nova Letra, 2007.

11 - Cole TJ, Bellizzi MC, Flegal KM, Dietz WH. Establishing a standard definition for child overweight and obesity worldwide: international survey. BMJ 2000; 320: 1240-3.

12 - PNDS. Pesquisa Nacional sobre Demografia e Saúde 1996. Relatório Preliminar. Rio de Janeiro; 1996.

13 - Assis AMO, Barreto ML. Condições de vida, saúde e nutrição na infância em Salvador. Salvador: UFBA; 2000.

14 - Corso ACT, Botelho LJ, Zeni LAZR, Moreira EAM. Sobrepeso em crianças menores de 6 anos de idade em Florianópolis, SC. Rev. Nutr. 2003, vol. 16, no. 1.

15 - WHO.World Health Organization. Global Database on Child Growth and Malnutrition. Program of Nutrition Family and Reproductive Health. Geneva; 1997.

16 - Waterlow JC. Causes and mechanisms of linear growth retardation (stunting). Eur J Nutr 1994; 48 (suppl.1):S1-4.

17 - Bundred P, Kitchiner D, Buchan I. Prevalence of overweight and obese children between 1989 and 1998: population based series of cross sectional studies. BMJ 2001; 322: 326-8.

18 - Canning PM, Courage ML, Frizzell LM. Prevalence of overweight and obesity in a provincial population of Canadian preschool children. CMAJ 2004; 171(3): 240-2.

Recebido para publicação em 27/12/2007.

Aprovado para publicação em 12/05/2008. 